\title{
COMPARATIVE STUDY OF STABILIZATION CONTROLS OF A FORKLIFT VEHICLE
}

\author{
Augie WIDYOTRIATMO* \\ *Faculty of Industrial Technology, Instrumentation and Control Research Group, \\ Insititut Teknologi Bandung, Ganesa 10, Bandung 40132, Indonesia \\ augie@tf.itb.ac.id \\ received 7 January 2019, revised 26 September, accepted 30 September
}

\begin{abstract}
This paper presents the control designs for an autonomous forklift vehicle that drive the vehicle from an initial configuration to a final one. Three stabilization controls, which are chained-form time-varying control, sigma-transformed discontinuous control, and navigation-variables-based discontinuous control, for a forklift vehicle are compared by simulations. The sigma-transformed and navigation-variables-based discontinuous controls provide fast convergence motions from an initial to a final configuration, while the time-varying-based control provides oscillatory motion and slow convergence. The sigma-transformed discontinuous control has a set of discontinuous points in which, from a practical point of view, the control signals can blow up if a vehicle enters the set. The navigation-variables-based control, which also has a discontinuous point at the final configuration, does not produce blown up control signals since its boundedness nature. Discussion on the implementation of control algorithm is elucidated for the three stabilization controls for the forklift vehicle.
\end{abstract}

Key words: Autonomous vehicle, stabilization control, time-varying-based control, discontinuous control, navigation-variables-based control

\section{INTRODUCTION}

Control design of autonomous vehicle is still of interest to many researchers, especially in dealing with implementation (Kłosiński et al., 2015; Virgalaivan et al., 2018; Widyotriatmo and Hong, 2015; Baranowski and Siwek, 2018). One of the applications is the stabilization control, which is the design of control algorithm to drive an autonomous vehicle from an initial configuration to a final configuration. The configuration is not only the position but also the orientation of the vehicle.

A wheeled vehicle is a nonholonomic system (i.e., a system with nonholonomic constraints). Brockett (1983) showed that there is no continuously differentiable time-invariant control law that stabilizes a nonholonomic system asymptotically. Two control strategies for nonholonomic systems are found in the literature: open loop and closed loop strategies. In open loop strategies (Murray and Sastry, 1993; Soueres and Laumond, 1996; Widyotriatmo and Hong, 2008), the control signals are calculated off-line using the information of the initial and the goal configurations. Using this strategy, the vehicle may not reach the goal configuration due to disturbances, modelling errors, and sensor uncertainties. In closed loop strategies, the control signals are computed online based on the current and goal configurations. A coordinate transformation is used. Examples of coordinate transformation are the chained form (Murray and Sastry, 1993), the power form (Pomet and Samson, 1994), the polar coordinates (Aicardi et al., 1995), the sigma process (Astolfi, 1996), the differential flatness approach (Lamiraux and Laumond, 2000; Tang et al., 2008; Ryu and Agarwal, 2010), and the transverse form (Morin and Samson, 2009). Several control algorithms proposed are the time-varying control (Samson, 1995; Tamba et al., 2009; Wang et al., 2015), discontinuous control (Lamiraux and Laumond, 2000), and the switching method (Hespanha and
Morse, 1999). Control laws using polar coordinates are utilized (Widyotriatmo and Hong, 2015; Astolfi, 1996; Siegwart and Nourbakhsh, 2004). Unlike the previous studies, which considered unicycle-type vehicles, the stabilization control for forklift vehicle is focused.

In dealing with the stabilization of nonholonomic wheeled mobile robots system, the system needs to be transformed into a special structure as in Abbasi et al. (2019), the power form (Xie and Li, 2019), chained form (S'anchez-Torres et al., 2019), or the polar or navigation-variable form (Widyotriatmo and Hong, 2012). Then, the control designs are implemented in the forms of timevarying or discontinuous controls. In Widyotriatmo and Hong (2012), experimental results for the stabilization of forklift vehicle using the navigation-variable form transformation has been performed. Other experimental results of a car-like-vehicle using a discontinuous control has been shown in Hashimoto et al. (2019). A switched pivot and longitudinal motions are also performed for point stabilization for a differential wheel robot with inverted pendulum Yue et al. (2019). Lyapunov method is established for the stabilization of mobile robot with inverted pendulum in Muralidharan and Mahindrakar (2014). Simultaneous stabilization and tracking of a nonholonomic robot using Lyapunov based approach is proposed in Wand et al. (2015). In Muralidharan and Mahindrakar (2013), the stabilization of mobile robot with constraints of configuration is solved by sliding mode technique. Stabilization of a mobile robot using model predictive control is presented in Xiao et al. (2017), Wei et al. (2013).

Different transformations and control strategies claim the effectiveness of the stabilization result. The question leads to how the control performances of the individual strategies of transformed systems for the forklift vehicle are. In this paper, the performances of the three configuration control laws, which are the time-varying-based control in the chained-form, the discontinuous 
control using sigma process, and the navigation-variables-based control, for asymptotically stabilizing the configuration error of the vehicle are compared. The trajectories calculated using the individual control laws are presented by simulations. From this simulation, the use of the three configuration control laws is analysed. The objective of this paper is to determine which transformation and control strategy are the most effective in stabilizing a forklift vehicle, focusing on the ease of implementation and on the resulted motion in driving the forklift from an initial configuration to a final one. By studying the comparisons of different transformation and control strategies, the engineer who applies the stabilization control of a forklift vehicle can understand the consequences in implementing a chosen control algorithm.

The equations of motion of the considered forklift are derived. The equations are represented kinematic and dynamic equations. The kinematic equations describe the velocity of the vehicle with the inputs of linear velocity and steering angle, and the dynamic equations provide the accelerations of the vehicle and the torques of the actuators of driving and steering. The control problem is divided into two stages. First, the linear velocity and the steering angle of the kinematic equations are designed and become the references for the dynamic model. And then, the input to two actuators (driving and steering) are calculated in such a way that the two actuators track the linear velocity and the steering angle.

The rest of this paper is organized as follows. The equations of motion of the vehicle are derived in Section II. The implementation of the PD control for the direct current (DC) motor control is discussed in Section III. Section IV presents the three configuration control laws for the forklift. Section $V$ shows the simulation results and discussion. The conclusions are drawn in Section VI.

\section{EQUATIONS OF MOTION OF FORKLIFT VEHICLE}

Let the global coordinate frame $O-\hat{\imath} \hat{j}$ be fixed in the workspace as in Fig. 1 . The body coordinate is denoted as $O_{\mathrm{b}}-\hat{\imath}_{\mathrm{b}} \hat{\jmath}_{\mathrm{b}}$, $l$ is the length from the rear wheel to $O_{\mathrm{b}}$. The position of the vehicle is $(x, y)$ located at $O_{\mathrm{b}}$ and the orientation $\theta$. The linear velocity and steering angle are denoted by $v$ and $\delta$, respectively.

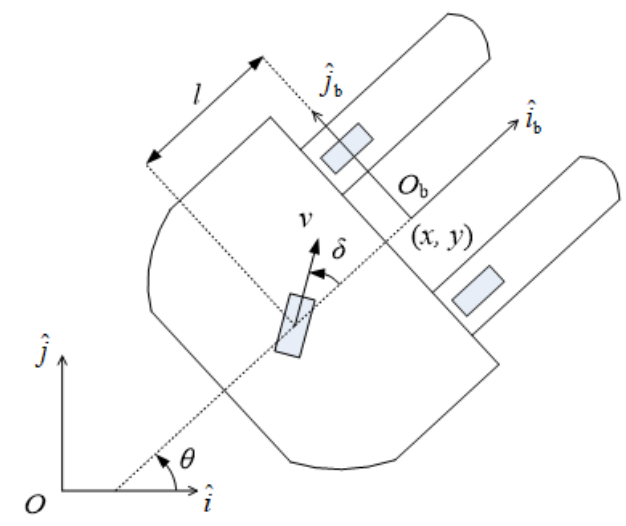

Fig. 1. The schematic of forklift vehicle

The angular positions of the front and rear wheels are denoted by $\varphi_{\mathrm{f}}$ and $\varphi_{\mathrm{b}}$, respectively. Let $\mathrm{q}$ be the state vector $\mathrm{q}=$ $\left[x, y, \theta, \delta, \varphi_{f}, \varphi_{b}\right]^{T} \in R^{6}$. The equations of motion of the forklift vehicle are:

$$
\begin{aligned}
& \mathrm{M}(\mathrm{q}) \ddot{\mathrm{q}}+\mathrm{C}(\mathrm{q}, \dot{\mathrm{q}}) \dot{\mathrm{q}}+\mathrm{f}_{s}+\mathrm{g}(\mathrm{q})=\mathrm{B}(\mathrm{q}) \tau+\mathrm{J}(\mathrm{q}) \mathrm{f}_{C}, \\
& \mathrm{~J}^{T}(\mathrm{q}) \dot{\mathrm{q}}=0,
\end{aligned}
$$

where: $\mathrm{M}(\mathrm{q}) \in R^{6 \times 6}$ is the inertia matrix, $\mathrm{C}(\mathrm{q}, \dot{\mathrm{q}}) \in R^{6 \times 6}$ is the centripetal and Coriolis matrix, $\mathrm{f}_{s} \in R^{6}$ is the surface friction vector, $\mathrm{g}(\mathrm{q}) \in R^{6}$ is the gravitational vector, $\mathrm{B}(\mathrm{q}) \in R^{6 \times 2}$ is the input matrix, $\tau=\left[\tau_{\mathrm{v}}, \tau_{\delta}\right]^{T} \in R^{2}$ is the torque inputs vector of the driving and steering motors, respectively, $\mathrm{f}_{C} \in R^{4}$ is the force vector denoting the constraint, and $\mathrm{J}(\mathrm{q}) \in R^{6 \times 4}$ is the constraint. Since the vehicle moves on the flat surface $g(q)=0$.

For non-slipping and pure rolling conditions, the constraint matrix $\mathrm{J}(\mathrm{q})$ is:

$\mathrm{J}(\mathrm{q})=\left[\begin{array}{llll}J_{11} & J_{12} & J_{13} & J_{14} \\ J_{21} & J_{22} & J_{23} & J_{24} \\ J_{31} & J_{32} & J_{33} & J_{34} \\ J_{41} & J_{42} & J_{43} & J_{44} \\ J_{51} & J_{52} & J_{53} & J_{54} \\ J_{61} & J_{62} & J_{63} & J_{64}\end{array}\right]$,

where: $J_{11}=-\sin \theta, \quad J_{12}=-(\cos \theta \sin \delta+\sin \theta \cos \delta)$, $J_{13}=\cos \theta, \quad J_{14}=\cos \theta \cos \delta-\sin \theta \sin \delta, \quad J_{21}=\cos \theta$, $J_{22}=\cos \theta \cos \delta-\sin \theta \sin \delta, \quad J_{23}=\sin \theta, \quad J_{24}=$ $\cos \theta \sin \delta+\sin \theta \cos \delta, \quad J_{32}=-l \cos \delta, J_{34}=-l \sin \delta$, $J_{53}=-r_{f}, \quad J_{64}=-r_{r}$, and $J_{31}=J_{32}=J_{33}=J_{34}=J_{51}=$ $J_{52}=J_{54}=J_{61}=J_{62}=J_{63}=0, r_{f}$ and $r_{r}$ are the radii of the wheels of the vehicle, at the front and rear. A matrix $\mathrm{S}(\mathrm{q}) \in R^{6 \times 2}$ is defined as:

$\mathrm{S}(\mathrm{q})=\left[\begin{array}{llllll}S_{11} & S_{12} & S_{13} & S_{14} & S_{15} & S_{16} \\ S_{21} & S_{22} & S_{23} & S_{24} & S_{25} & S_{26}\end{array}\right]^{T}$,

where: $\quad S_{11}=\cos \theta \cos \delta, \quad S_{11}=\sin \theta \cos \delta, \quad S_{13}=$ $-(1 / l) \sin \delta, S_{15}=\left(1 / r_{\mathrm{f}}\right) \cos \delta, S_{16}=1 / r_{r}, S_{24}=1$, and $S_{21}=S_{22}=S_{23}=S_{25}=S_{26}=0$, such that $\mathrm{S}(\mathrm{q})^{T} \mathrm{~J}(\mathrm{q})=0$. The velocity vector $\mathrm{v}=\left[\begin{array}{ll}v & \dot{\delta}\end{array}\right]^{T}$ is defined where $v$ is the linear velocity and the angular velocity $\dot{\delta}$. The derivative of $\mathrm{q}$ with respect to time is:

$\dot{\mathrm{q}}=\mathrm{S}(\mathrm{q}) \mathrm{v}$,

Lagrange method is used to obtain (1). The kinetic energy $K$ is:

$K=\frac{1}{2} \dot{\mathrm{q}}^{\mathrm{T}} \mathrm{M} \dot{\mathrm{q}}$,

where:

$\mathrm{M}=\operatorname{diag}\left(m, m, I_{\mathrm{b}}, I_{\delta}, I_{\mathrm{f}}, I_{\mathrm{r}}\right)$,

$m$ is the vehicle's mass, $I_{\mathrm{b}}$ is the moment of inertia around $O_{\mathrm{b}}, I_{\delta}$ is the moment of inertia of the rear wheel towards its normal axis, and $I_{\mathrm{f}}$ and $I_{\mathrm{r}}$ are the front and rear wheels' moments of inertia. The centripetal and Coriolis matrix is:

$\mathrm{C}(\mathrm{q}, \dot{\mathrm{q}})=\dot{\mathrm{M}}(\mathrm{q}) \dot{\mathrm{q}}-\partial K / \partial \mathrm{q}=0$,

and the input transformation $B(q)$ is:

$\mathrm{B}(\mathrm{q})=\left[\begin{array}{llllll}0 & 0 & 0 & 0 & 0 & 1 \\ 0 & 0 & 0 & 1 & 0 & 0\end{array}\right]^{T}$.

The second derivative of vector $\mathrm{q}$ is:

$\ddot{\mathrm{q}}=\mathrm{S}(\mathrm{q}) \dot{\mathrm{v}}+\dot{\mathrm{S}}(\mathrm{q}, \dot{\mathrm{q}}) \mathrm{v}$. 
Substituting (10) into (1), and multiplying by $S^{T}(\mathrm{q})$ of both sides, it is obtained:

$\mathrm{M}_{1}(\mathrm{q}) \dot{\mathrm{v}}+\mathrm{C}_{1}(\mathrm{q}, \dot{\mathrm{q}}) \mathrm{v}+\mathrm{f}_{1}=\mathrm{B}_{1}(\mathrm{q}) \tau$,

where:

$\mathrm{M}_{1}(\mathrm{q})=\mathrm{S}^{T}(\mathrm{q}) \mathrm{M}(\mathrm{q}) \mathrm{S}(\mathrm{q})$,

$\mathrm{C}_{1}(\mathrm{q}, \dot{\mathrm{q}})=\mathrm{S}^{T}(\mathrm{q}) \mathrm{M}(\mathrm{q}) \dot{\mathrm{S}}(\mathrm{q})$,

$\mathrm{f}_{1}=\mathrm{S}^{T}(\mathrm{q}) \mathrm{f}=\left[\begin{array}{ll}f_{v} & f_{\delta}\end{array}\right]^{T}$,

$\mathrm{B}_{1}(\mathrm{q})=\operatorname{diag}\left(1 / r_{\mathrm{r}}, 1\right)$,

$f_{v}$ and $f_{\delta}$ are the surface frictions in the directions of linear velocity and steering angle of the rear wheel. Then, the equations of motion are:

$\dot{x}=v \cos \theta \cos \delta$,

$\dot{y}=v \sin \theta \cos \delta$,

$\dot{\theta}=-(v / l) \sin \delta$,

$m_{1} \dot{v}+c_{1} v+r_{\mathrm{r}} f_{v}=\tau_{v}$

$I_{\delta} \ddot{\delta}+f_{\delta}=\tau_{\delta}$,

where:

$m_{1}=r_{\mathrm{r}}\left(\left(m+I_{\mathrm{f}}\right)(\cos \delta)^{2}+\left(I_{\mathrm{b}} / l^{2}\right)(\sin \delta)^{2}+I_{\mathrm{r}}\right)$,

$c_{1}=r_{\mathrm{r}}\left(\left(I_{\mathrm{b}} / l^{2}\right)-\left(I_{\mathrm{f}} / r_{\mathrm{f}}^{2}\right)-m\right) \dot{\delta} \cos \delta \sin \delta$.

The equations (16)-(18) show the kinematic equations, (19) and (20) are the traction and steering dynamics, respectively.

\section{MOTOR CONTROL}

The rear wheel in (19)-(20) are controlled by the torques $\tau_{v}$ and $\tau_{\delta}$ as:

$\tau_{v}=\left(k_{\mathrm{m}, v} / R_{\mathrm{m}, v}\right)\left(\left(u_{v}-\left(k_{\mathrm{emf}, v} / r_{r}\right) v\right)\right.$,

$\tau_{\delta}=\left(k_{\mathrm{m}, \delta} / R_{\mathrm{m}, \delta}\right)\left(u_{\delta}-k_{\mathrm{emf}, \delta} \dot{\delta}\right)$,

where: $u_{v}$ and $u_{\delta}$ are the control signals for the driving and steering motors. $k_{\mathrm{m}, i}$ is the motor torque constant, $k_{\mathrm{emf}, i}$ is the back electromotive constant, and $R_{\mathrm{m}, i}$ is the motor resistance, of the $i$ th motor, $i=v, \delta$.

The desired linear velocity $v_{d}$ and steering angle $\delta_{d}$ is controlled by the PD with control signals $u_{v}$ and $u_{\delta}$ are designed as:

$u_{v}=k_{\mathrm{P}, v}\left(v_{d}-v\right)-k_{\mathrm{D}, v} \dot{v}$,

$u_{\delta}=k_{\mathrm{P}, \delta}\left(\delta_{d}-\delta\right)-k_{\mathrm{D}, \delta} \dot{\delta}$,

where: $k_{\mathrm{P}, i}$ is the proportional gain, $k_{\mathrm{D}, i}$ is the derivative gain of the $i$-th motor, $i=v, \delta$. Using (23) and (25), (19) becomes

$\left(\frac{R_{\mathrm{m}, v} m_{1}}{k_{\mathrm{m}, v}}+k_{\mathrm{D}, v}\right) \dot{v}+\left(\frac{R_{\mathrm{m}, v} c_{1}}{k_{\mathrm{m}, v}}+\frac{k_{\mathrm{em}, v}}{r_{r}}+k_{\mathrm{P}, v}\right) v=k_{\mathrm{P}, v} v_{d}-$ $\frac{R_{\mathrm{m}, v} r_{r}}{k_{\mathrm{m}, v}} f_{v}$.

By choosing $k_{\mathrm{P}, v}>\max \left(\left(R_{\mathrm{m}, v} / k_{\mathrm{m}, v}\right) c_{1}-k_{\mathrm{emf}, v} / r_{r}\right)$ and assuming $f_{v}$ is constant, the solution of (27) is obtained as: $v(t)=\frac{k_{p, v}}{\frac{\left(c_{1} R_{m, v}\right)}{k_{m, v}}+\frac{k_{\mathrm{emf}, v}}{r_{r}}+k_{p, v}}\left(v_{\mathrm{d}}-\frac{R_{m, v} r_{r}}{k_{m, v} k_{p, v}} f_{v}-\left(v_{d}-\right.\right.$
$\left.v\left(t_{0}\right)-\frac{R_{m, v} v_{r}}{k_{m, v} k_{p, v}} f_{v}\right) \exp \left(-\lambda_{v} t\right)$,

where:

$\lambda_{v}=\frac{\left(\frac{c_{1} R_{\mathrm{m}, v}}{k_{\mathrm{m}, v}}+\frac{k_{\mathrm{emf}, v}}{r_{r}}+k_{\mathrm{P}, v}\right)}{\left(\frac{m_{1} R_{\mathrm{m}, v}}{k_{\mathrm{m}, v}}+k_{\mathrm{D}, v}\right)}>0$.

Using (24) and (26), (20) becomes:

$\left(\frac{I_{\delta} R_{\mathrm{m}, \delta}}{k_{\mathrm{m}, \delta} k_{\mathrm{P}, \delta}}\right) \ddot{\delta}+\left(\frac{k_{\mathrm{D}, \delta}+k_{\mathrm{emf}, \delta}}{k_{\mathrm{P}, \delta}}\right) \dot{\delta}+\delta=\delta_{d}-\left(\frac{R_{\mathrm{m}, \delta}}{k_{\mathrm{m}, \delta} k_{\mathrm{P}, \delta}}\right) f_{\delta}$.

The parameters $k_{\mathrm{P}, \delta}$ and $k_{\mathrm{D}, \delta}$ are chosen such that:

$\left(\left(k_{\mathrm{D}, \delta}+k_{\mathrm{emf}, \delta}\right) / k_{\mathrm{P}, \delta}\right)^{2}-4\left(R_{\mathrm{m}, \delta} I_{\delta}\right) /\left(k_{\mathrm{m}, \delta} k_{\mathrm{P}, \delta}\right)>0$.

The solution of (30) becomes:

$\delta(t)=\delta_{\mathrm{d}}-\frac{R_{\mathrm{m}, \delta}}{k_{\mathrm{m}, \delta} k_{\mathrm{P}, \delta}} f_{\delta}-\left(\delta_{\mathrm{d}}-\delta\left(t_{0}\right)-\frac{R_{m, \delta}}{k_{m, \delta} k_{P, \delta}} f_{\delta}\right) \times$
$\left(\frac{\lambda_{\delta, 2} \exp \left(-\lambda_{\delta, 1} t\right)-\lambda_{\delta, 1} \exp \left(-\lambda_{\delta, 2} t\right)}{\lambda_{\delta, 2}-\lambda_{\delta, 1}}\right)$,

where:

$\lambda_{\delta, 1}=\frac{\frac{k_{D, \delta}+k_{\mathrm{emf}, \delta}}{k_{P, \delta}}+\left(\left(\frac{k_{D, \delta}-k_{\mathrm{emf}, \delta}}{k_{P, \delta}}\right)^{2}-\frac{4 R_{m, \delta} I \delta}{k_{m, \delta^{k}}{ }^{2} \delta}\right)^{\frac{1}{2}}}{2 \frac{R_{m, \delta} \delta}{k_{m, \delta} k_{P, \delta}}}>0$,

$\lambda_{\delta, 2}=\frac{\frac{k_{D, \delta}+k_{\mathrm{emf}, \delta}}{k_{P, \delta}}-\left(\left(\frac{k_{D, \delta}-k_{\mathrm{emf}, \delta}}{k_{P, \delta}}\right)^{2}-\frac{4 R_{m, \delta} I_{\delta} \delta}{k_{m, \delta^{k}, \delta} k_{P, \delta}}\right)^{\frac{1}{2}}}{2 \frac{R_{m, \delta} \delta^{I} \delta}{k_{m, \delta^{k}, \delta}}}>0$.

It can be seen that in (28) and (32), the PD control can make the linear velocity $v$ and the steering angle $\delta$ to track the desired values $v_{d}$ and $\delta_{d}$ with some errors caused by the frictions and the decaying exponential function. It is assumed that the friction is very small and so that the PD control can track the desired values.

\section{STABILIZATION CONTROLS}

By assuming the ideal linear velocity and steering angle as inputs $\left(v=v_{\mathrm{d}}, \delta=\delta_{\mathrm{d}}\right)$, the configuration control algorithms for the forklift based on the time-varying-based control using chainedform with time-varying control, sigma-transformed discontinuous control, and navigation-variables-based control are investigated. Without loss of generality, the goal configuration is set to $(\mathrm{gg}, \mathrm{yg}$, $\left.\theta_{\mathrm{g}}\right)=(0,0,0)$.

Let $v_{1}=v \cos \delta$ and $v_{2}=v \sin \delta$. In terms of $v_{1}$ and $v_{2}$, The linear velocity and steering angle are calculated as follows:

$v=\operatorname{sgn}\left(v_{1}\right) \sqrt{v_{1}^{2}+v_{2}^{2}}$,

$\delta=\arctan \left(v_{2} / v_{1}\right)$.

The kinematic equations in (16)-(18) becomes:

$\dot{x}=v_{1} \cos \theta$, 
$\dot{V}_{c}=-k_{v, 1} \chi_{3}^{\prime 2} \leq 0$.

$\dot{y}=v_{1} \sin \theta$,

$\dot{\theta}=-v_{2} / l$.

Let $\tilde{x}=x_{g}-x, \tilde{y}=y_{g}-y$, and $\tilde{\theta}=\theta_{g}-\theta$ be the errors between the current and final configurations. Using the kinematic equations in (37)-(39), the individual transformations, which are the chain form for deriving the time-varying-based control, the sigma process for calculating the discontinuous control, and the navigation-variables for obtaining the navigation-variables-based control are introduced in the following subsections.

\subsection{Chained-form time-varying-based control}

In deriving the time-varying-based control, the following chained-form space $\left(\chi_{1}^{\prime}, \chi_{2}^{\prime}, \chi_{3}^{\prime}\right)$ is introduced:

$\chi_{1}^{\prime}=\tilde{\theta}$,

$\chi_{2}^{\prime}=\tilde{x} \sin \tilde{\theta}-\tilde{y} \cos \tilde{\theta}$,

$\chi_{3}^{\prime}=\tilde{x} \cos \tilde{\theta}+\tilde{y} \sin \tilde{\theta}$,

$v_{1}^{\prime}=-\left(v_{2} / l\right)$,

$v_{2}^{\prime}=v_{1}-\chi_{2}^{\prime} v_{2} / l$.

From (40)-(42), the origins of the state of the chained-form space $\left(\chi_{1}^{\prime}, \chi_{2}^{\prime}, \chi_{3}^{\prime}\right)$ and the configuration error space $(\tilde{x}, \tilde{y}, \tilde{\theta})$ coincide. Thus, the asymptotical stabilization problem of the configuration error space becomes that of the chained-form space. The chained-form satisfies the following:

$\dot{\chi}_{1}^{\prime}=v_{1}^{\prime}$,

$\dot{\chi}_{2}^{\prime}=\chi_{3} v_{1}^{\prime}$,

$\dot{\chi}_{3}^{\prime}=v_{2}^{\prime}$.

The control law for stabilizing the chained-form of (45)-(47) is declared in Theorem 1.

Theorem 1: Consider the chained form system (45)-(47). Let the control law $v_{1}^{\prime}$ and $v_{2}^{\prime}$ be designed as

$v_{1}^{\prime}=-k_{v, 1} \chi_{1}^{\prime}+\left(\chi_{2}^{\prime 2}+\chi_{3}^{\prime 2}\right) \sin t$

$v_{2}^{\prime}=-\chi_{2}^{\prime} v_{1}^{\prime}-k_{v, 2} \chi_{3}^{\prime}$

where $k_{v, 1}$ and $k_{v, 2}$ are positive constants. Then, the origin $\left(\chi_{1}^{\prime}\right.$, $\left.\chi_{2}^{\prime}, \chi_{3}^{\prime}\right)=(0,0,0)$ is asymptotically stable.

Proof: Using (48)-(49), (45)-(47) becomes:

$\dot{\chi}_{1}^{\prime}=-k_{v, 1} \chi_{1}^{\prime}+\left(\chi_{2}^{\prime 2}+\chi_{3}^{\prime 2}\right) \sin t$

$\dot{\chi}_{2}^{\prime}=-k_{v, 1} \chi_{1}^{\prime} \chi_{3}^{\prime}+\left(\chi_{2}^{\prime 2}+\chi_{3}^{\prime 2}\right) \chi_{3}^{\prime} \sin t$,

$\dot{\chi}_{3}^{\prime}=-k_{v, 2} \chi_{3}^{\prime}+k_{v, 1} \chi_{1}^{\prime} \chi_{2}^{\prime}-\left(\chi_{2}^{\prime 2}+\chi_{3}^{\prime 2}\right) \chi_{2}^{\prime} \sin t$.

Note that $\left(\chi_{2}^{\prime 2}+\chi_{3}^{\prime 2}\right) \sin t$ is a time-varying function that satisfies $\left(\chi_{2}^{\prime 2}+\chi_{3}^{\prime 2}\right) \sin t=0$ as $\chi_{2}^{\prime}, \chi_{3}^{\prime}=0$.

From (50), $\chi_{1}^{\prime}$ is bounded, which implies that $v_{1}^{\prime}$ is also bounded. The Lyapunov function $V_{c}$ is defined as:

$V_{c}=\chi_{2}^{\prime 2}+\chi_{3}^{\prime 2}$.

Using (51)-(52), the time-derivative of the Lyapunov function (53) becomes:
This implies that $\chi_{3}^{\prime} \rightarrow 0$ as $t \rightarrow \infty$. Then, from (51) and (52), it can be concluded that $\chi_{2}^{\prime} \rightarrow 0$ as $t \rightarrow \infty$. Since $\left(\chi_{2}^{\prime 2}+\right.$ $\left.\chi_{3}^{\prime 2}\right) \sin t=0$ as $\chi_{2}^{\prime}, \chi_{3}^{\prime}=0, v_{1}^{\prime}$ tends to zero. Then from (48), it can be concluded that $\chi_{1}^{\prime} \rightarrow 0$ as $t \rightarrow \infty$. Thus, it is obtained that $\chi_{1}^{\prime}, \chi_{2}^{\prime}, \chi_{3}^{\prime} \rightarrow 0$ as $t \rightarrow \infty$.

\subsection{Sigma-transformed discontinuous-based control}

The sigma-transformed discontinuous-based control is derived using the following transformation:

$\chi_{1}^{\prime \prime}=\tilde{x}$,

$\chi_{2}^{\prime \prime}=\tilde{y} / \tilde{x}$

$\chi_{3}^{\prime \prime}=\tilde{\theta}$,

$v_{1}^{\prime \prime}=v_{1} \cos \tilde{\theta}$

$v_{2}^{\prime \prime}=-v_{2} / l$.

As in the chained form, the origins of the state of the sigma transformation space $\left(\chi_{1}^{\prime \prime}, \chi_{2}^{\prime \prime}, \chi_{3}^{\prime \prime}\right)$ and the configuration error space $(\tilde{x}, \tilde{y}, \tilde{\theta})$ also coincide. The transformed system is described by:

$\dot{\chi}_{1}^{\prime \prime}=v_{1}^{\prime \prime}$,

$\dot{\chi}_{2}^{\prime \prime}=v_{1}^{\prime \prime}\left(\tan \chi_{3}^{\prime \prime}-\chi_{2}^{\prime \prime}\right) / \chi_{1}^{\prime \prime}$,

$\dot{\chi}_{3}^{\prime \prime}=v_{2}^{\prime \prime}$.

The control law of the sigma-transformed discontinuous-based control is expressed in Theorem 2.

Theorem 2: Consider the system in (60)-(62). Let the control law $v_{1}^{\prime \prime}$ and $v_{2}^{\prime \prime}$ be

$v_{1}^{\prime \prime}=-k_{v, 1} \chi_{1}^{\prime \prime}$,

$v_{2}^{\prime \prime}=k_{v, 2} \chi_{2}^{\prime \prime}+k_{v, 3} \chi_{3}^{\prime \prime}$,

where $k_{v, 1}, k_{v, 2}$, and $k_{v, 3}$ are the control parameters that are chosen in such a way that the matrix of linearized system have negative eigen-values. Then the origin of $\left(\chi_{1}^{\prime \prime}, \chi_{2}^{\prime \prime}, \chi_{3}^{\prime \prime}\right)$ is asymptotically stable.

Proof: The substitution of (63)-(64) into (60)-(62) yields:

$\dot{\chi}_{1}^{\prime \prime}=-k_{v, 1} \chi_{1}^{\prime \prime}$,

$\dot{\chi}_{2}^{\prime \prime}=-k_{v, 1}\left(\tan \chi_{3}^{\prime \prime}-\chi_{2}^{\prime \prime}\right)$,

$\dot{\chi}_{3}^{\prime \prime}=-k_{v, 2} \chi_{2}^{\prime \prime}-k_{v, 3} \chi_{3}^{\prime \prime}$.

By linearizing the system (66)-(67) around the origin, we obtain:

$\left[\begin{array}{c}\dot{\chi}_{2}^{\prime \prime} \\ \dot{\chi}_{3}^{\prime \prime}\end{array}\right]=A\left[\begin{array}{l}\chi_{2}^{\prime \prime} \\ \chi_{3}^{\prime \prime}\end{array}\right], A=\left[\begin{array}{cc}k_{v, 1} & -k_{v, 1} \\ -k_{v, 2} & -k_{v, 3}\end{array}\right]$

From (65), it is obvious that $\chi_{1}^{\prime \prime}$ goes to zero as time goes to infinity. If $k_{v, 1}>0, k_{v, 2}$ and $k_{v, 3}$ are designed such that the matrix $A$ has all the eigenvalues with negative real part, $\chi_{2}^{\prime \prime}, \chi_{3}^{\prime \prime}$ go to zero as time goes to infinity. Thus, the origin of $\left(\chi_{1}^{\prime \prime}, \chi_{2}^{\prime \prime}, \chi_{3}^{\prime \prime}\right)$ $=(0,0,0)$ is asymptotically stable. 


\subsection{Navigation-Variables-Based Control}

The navigation-variables-based control is classified as the discontinuous control. The coordinate of the forklift is to be transformed to specific variables, called navigation variables, as the distance error $(\rho)$, the orientation error component associated with the direction to the goal point $(\alpha)$, and the orientation error component associated with the direction of the goal frame $(\varphi)$. Note that $\tilde{\theta}=\alpha+\varphi$. In Fig. 2, the navigation variables are obtained as follows:

$\rho=\sqrt{\tilde{x}^{2}+\tilde{y}^{2}}$,

$\alpha=\arctan 2(\tilde{y}, \tilde{x})-\tilde{\theta}$,

$\varphi=\theta_{g}-\arctan 2(\tilde{y}, \tilde{x})$

The origin of navigation variables $(\rho, \alpha, \varphi)=(0,0,0)$ coincide with the origin of the error $(\tilde{x}, \tilde{y}, \tilde{\theta})=(0,0,0)$. Then, the kinematic equations in the introduced navigation variables become:

$\dot{\rho}=-v_{1} \cos \alpha$,

$\dot{\alpha}=\left(v_{1} / \rho\right) \sin \alpha+\left(v_{2} / l\right)$,

$\dot{\varphi}=-\left(v_{1} / \rho\right) \sin \alpha$.

The control law statement is established in Theorem 3.

Theorem 3: Consider the system (72)-(74). Let the control inputs $v_{1}$ and $v_{2}$ be designed as:

$v_{1}=k_{v, 1} \rho \cos \alpha$,

$v_{2}=\tan ^{-1}\left(-l\left(k_{v, 2} \alpha-k_{v, 2}(\varphi+\alpha) \frac{\cos \alpha \sin \alpha)}{\alpha}\right)\right.$.

where $k_{v, 1}$ and $k_{v, 2}$ are the positive gain constants. Then, the origin of navigation variables $(\rho, \alpha, \varphi)=(0,0,0)$ is asymptotically stable.

Proof: Substituting (75)-(76) into (72)-( 74) yields:

$\dot{\rho}=-k_{v, 1} \rho \cos ^{2} \alpha$,

$\dot{\alpha}=-k_{v, 2} \alpha-k_{v, 1}(\varphi / \alpha) \cos \alpha \sin \alpha$,

$\dot{\varphi}=-k_{v, 1} \cos \alpha \sin \alpha$.

Let a Lyapunov function $V_{n}$ be chosen as:

$V_{n}=(1 / 2)\left(\rho^{2}+\alpha^{2}+\varphi^{2}\right)$.

Using (77)-(79), the time-derivative of $V$ in (80) becomes:

$\dot{V}_{n}=-k_{v, 1} \rho^{2} \cos ^{2} \alpha-k_{v, 2} \alpha^{2} \leq 0$.

By Barbalat's Lemma, $\dot{V}_{n} \rightarrow 0$ as $t \rightarrow \infty$. This implies that the state $\rho, \alpha \rightarrow 0$ as $t \rightarrow \infty$. Let the set $S_{\text {nav }}=\{(\rho, \alpha, \varphi): \dot{V}(\rho, \alpha$, $\varphi)=0\}$. It can be seen from (78) that $\alpha(t) \equiv 0$ implies $\dot{\alpha}(t) \equiv$ 0 and leads to $\phi(t) \equiv 0$. Thus, there is no solution that can stay identically in $S_{\text {nav }}$, except the solutions $\rho, \alpha, \varphi=0$. Based on the LaSalle's Theorem, the origin $(\rho, \alpha, \varphi)=(0,0,0)$ is the largest invariant set and, thus, the origin is asymptotically stable.

\section{SIMULATION RESULTS AND DISCUSSIONS}

Here, the trajectories calculated by the three control laws, that is, time varying control, sigma-transformed discontinuous control, and navigation-variables-based control, for a forklift driven from an initial configuration $(-3,3,0)$ to a goal configuration $(0,0,0)$ are evaluated. The configuration of the vehicle is represented by $(x, y$, $\theta$ ) (where the unit of $x$ and $y$ is meter and that of $\theta$ is radian). It is assumed that the linear velocity and the steering angle can follow their control commands. Small disturbances that are assumed to be the imperfect tracking control in the dynamic model and the slippage in high-speed movement are included in the simulation.

The motion of the forklift using the time-varying-based control law (47)-(48) is shown in Fig. 3 and its trajectories $(x, y, \theta)$ are in Fig. 4. The gains are set to $k_{v, 1}=3 \mathrm{~s}^{-1}$ and $k_{v, 2}=1 \mathrm{~s}^{-1}$. The driving velocity $v_{\mathrm{d}}$ and steering angle $\delta_{d}$ are shown in Fig. 5 . It is shown in Figs. 3-5 that the time-varying control produces oscillating movements. The convergence rate of the configuration from the initial to the goal is very slow. In this simulation, the goal configuration was reached in $20 \mathrm{~s}$.

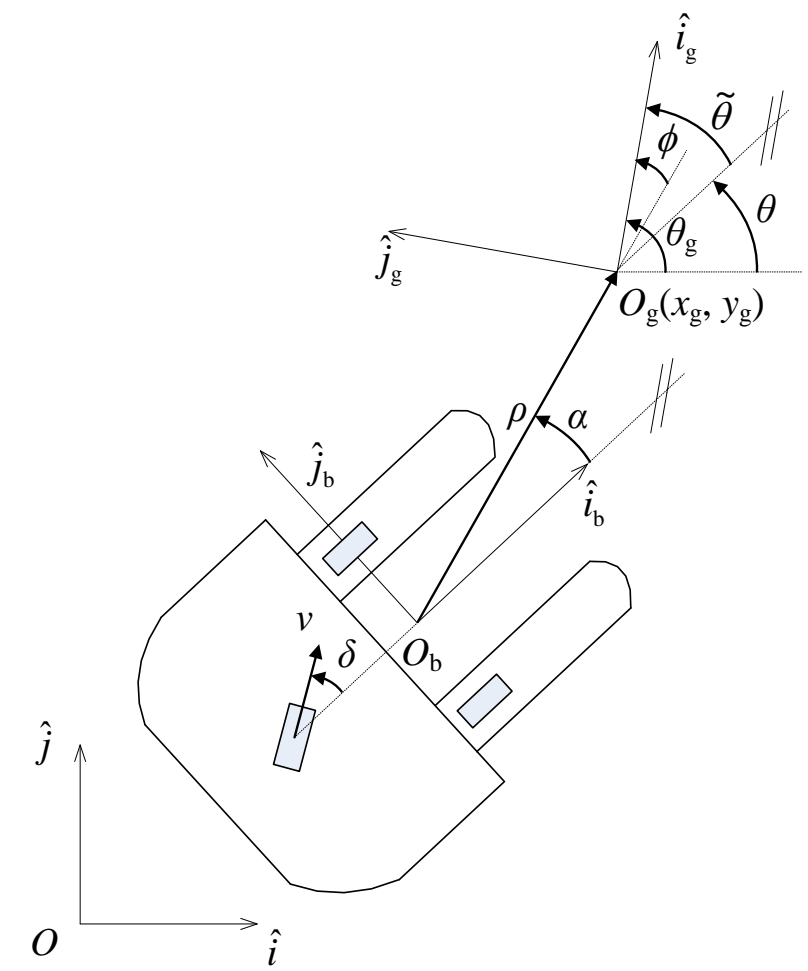

Fig. 2. The navigation variables $(\rho, \alpha, \varphi)$

The motion of the forklift using the discontinuous control law (62)-(63) with the gains $k_{v, 1}=1 \mathrm{~s}^{-1}$ and $k_{v, 2}=-4 \mathrm{~s}^{-1}, k_{v, 3}=3$ (the eigenvalues are all set to -1 ) that moves to the goal configuration is shown in Fig. 6. The configuration motion is depicted in Fig. 7. The driving velocity $v_{d}$ and steering angle $\delta_{d}$ are depicted in Fig. 8. It is observed that the sigma-transformed discontinuous control produces a natural and no oscillations motion. The convergence rate of the configuration from the initial to the goal is also faster than that of the time-varying control law.

In the sigma-transformed discontinuous control, the control signals are discontinues for all points along $x=0$. Therefore, the forklift is restricted to achieve the line $x=0$ since the control signals are not defined at that line. However, the forklift may approach the goal point $(x, y)=(0,0)$. The problem in the implementation of discontinuous control, the uncertainties from the sensors or actuators may lead the forklift to the situation where any point along $x=0$ is achieved by the forklift. Then, at this point, the control signals may not be determined since the sigma transfor- 
mation of (56) is not defined. Moreover, the control signals may be blown up when the vehicle achieve the points where $x$ is very close to zero. This can be seen from the transformation of $\chi_{2}^{\prime \prime}$ in (56) the control law $v_{2}^{\prime \prime}$ in (64).

The trajectories of the system calculated using the navigationvariables-based control (74)-(75) are investigated. The gains are set to $k_{v, 1}=1 \mathrm{~s}^{-1}$ and $k_{v, 2}=5 \mathrm{~s}^{-1}$. The motion of the forklift, the configuration, and the control inputs (driving velocity $v_{d}$ and steering angle $\delta_{d}$ ) are depicted in Figs. 9-11, respectively. As in the sigma-transformed discontinuous control law, the trajectories generated by the navigation-variables-based control renders a natural and no oscillation motion. The goal configuration was achieved in $6 \mathrm{~s}$.

The difference between the discontinuous control and the navigation-based control lies on the discontinuous points. The discontinuous points of the sigma-transformed discontinuous control are on all points along $x=0$, whereas the discontinuous point of the navigation-variables-based control is on one point that is $\rho=0$. In the navigation-variables-based control, the forklift may asymptotically converge to the goal point $\rho=0$, and accordingly $(\tilde{x}, \tilde{y})=(0$, $0)$. The gains of $k_{v, 1}$ and $k_{v, 2}$ can be adjusted so that the forklift approaching forward, or in other words, the angles $\alpha$ and $\varphi$ have already been zero. A switch control at the linear velocity $v=0$ can be applied at $\rho=0$, so that the forklift has arrived at the desired configuration and the movement is already completed.

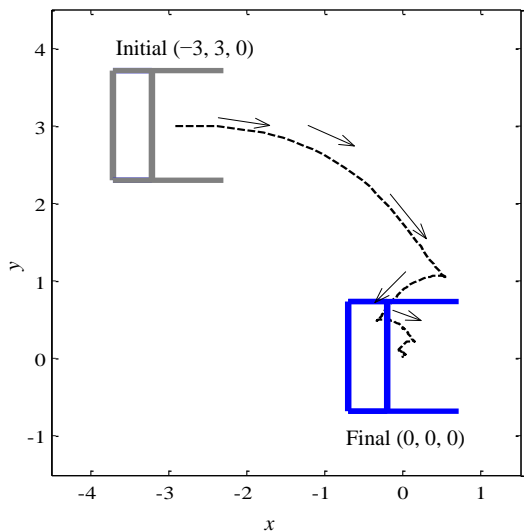

Fig. 3. Motion of the forklift using time-varying control law (48)-(49)

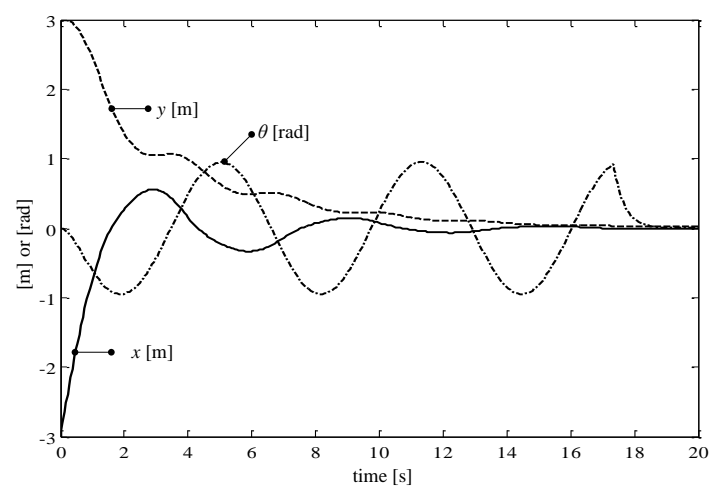

Fig. 4. Configuration motions $(x, y, \theta)$ using time-varying control law (48)-(49)

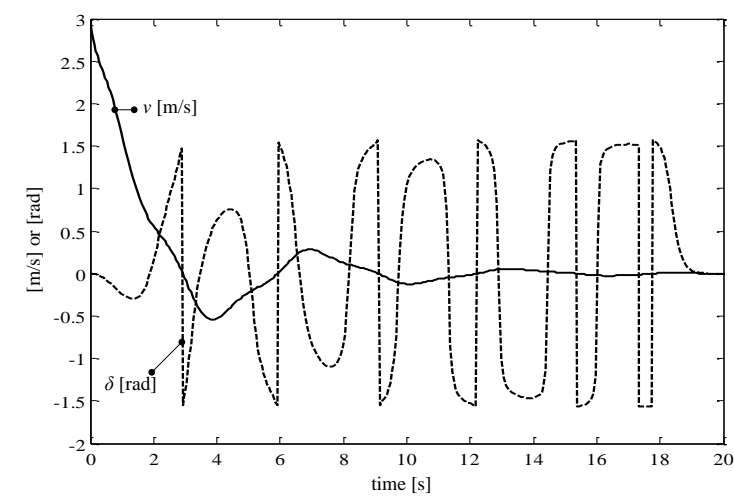

Fig. 5. Linear velocity $v$ and steering angle $\delta$ through time-varying control law (48)-(49)

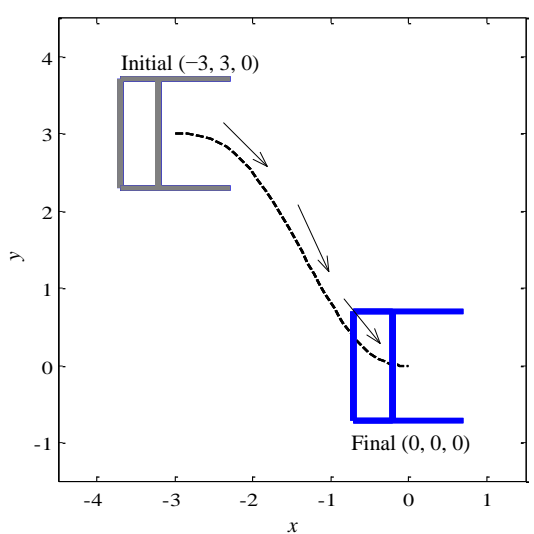

Fig. 6. Motion of the forklift using sigma transformed discontinuous control law (63)-(64)

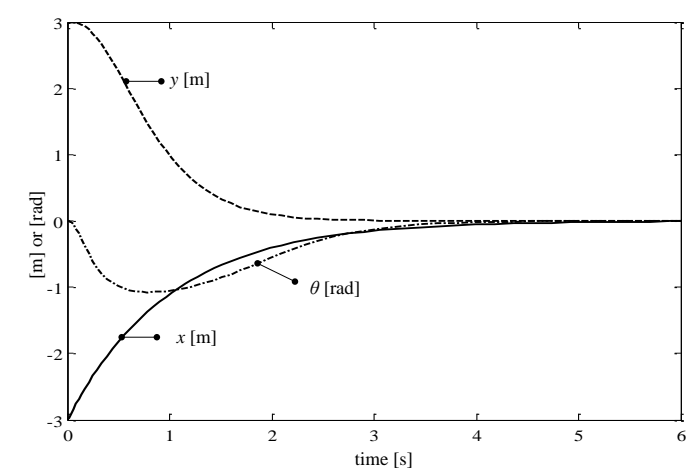

Fig. 7. Configuration motions $(x, y, \theta)$ using sigma transformed discontinuous control law (63)-(64)

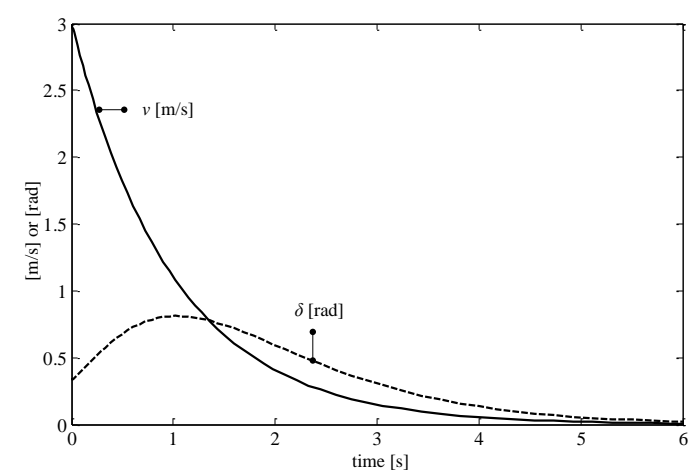

Fig. 8. Linear velocity $v$ and steering angle $\delta$ through sigma transformed discontinuous control law (63)-(64) 


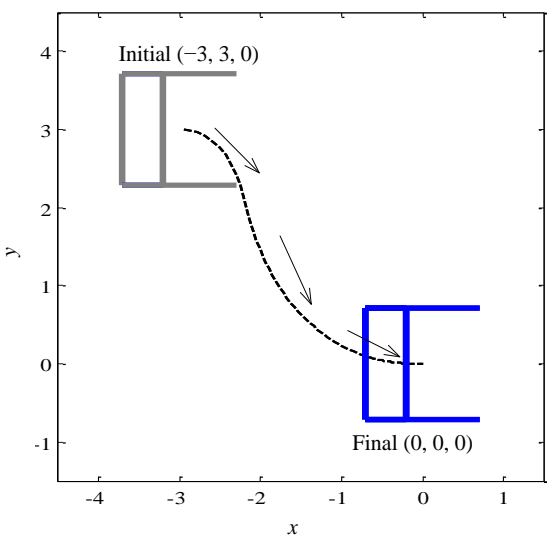

Fig. 9. Motion of the forklift using the navigation-variable-based control law (75)-(76)

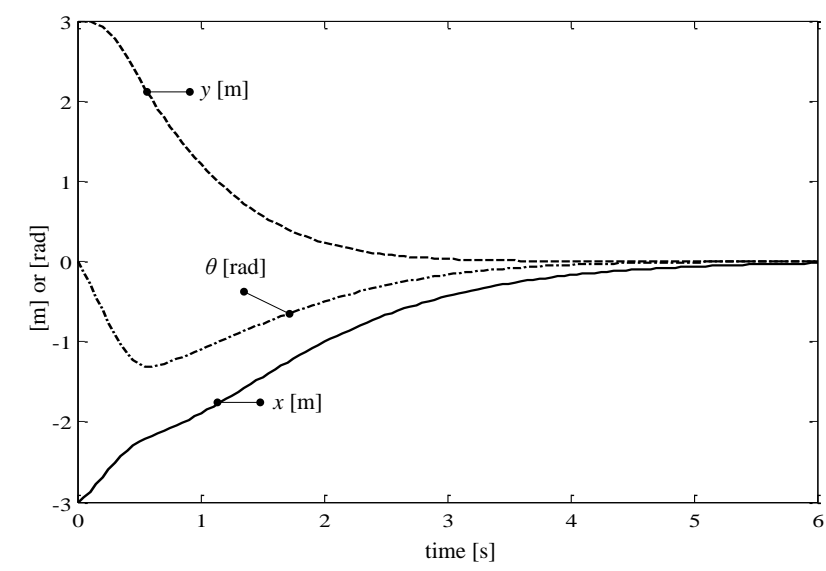

Fig. 10. The trajectories of $(x, y, \theta)$ using the navigation-variable-based control law (75)-(76)

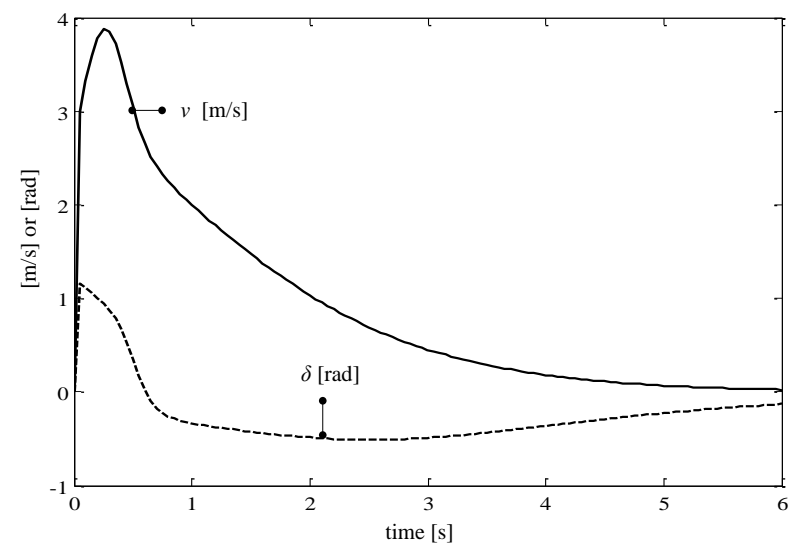

Fig. 11. Linear velocity $v$ and steering angle $\delta$ through navigation-variable-based control law (75)-(76)

One problem may occurs when the forklift uses the navigation-variables-based control and starts from the initial point $\rho=0$ or $(\tilde{x}, \tilde{y})=(0,0)$ but the orientation error $\tilde{\theta} \neq 0$. At this point, the navigation-variables-based control is not defined since (73) and (74) are no longer held. To cope with the problem, a check point where $\rho \neq 0$ should firstly be appointed, and the forklift shall be driven away from $\rho=0$. Then, the initial position changes to the check point where $\rho \neq 0$.
Tab. 1. Comparison of stabilization control of the chained-form, sigma-transformed, and the navigation-based controls

\begin{tabular}{|c|c|c|c|}
\hline \multirow{2}{*}{ Category } & \multicolumn{3}{|c|}{ Control algorithms } \\
\cline { 2 - 4 } & $\begin{array}{c}\text { Chained form } \\
\text { time-varying } \\
\text { control } \\
(48)-(49)\end{array}$ & $\begin{array}{c}\text { Sigma- } \\
\text { transformed } \\
\text { discontinuous } \\
\text { control (63)-(64) }\end{array}$ & $\begin{array}{c}\text { Navigation- } \\
\text { variables-based } \\
\text { discontinuous } \\
\text { control (75)-(76) }\end{array}$ \\
\hline Motion & Oscillation & No Oscillation & No Oscillation \\
\hline Convergence & Slow & Fast & Fast \\
\hline $\begin{array}{c}\text { Control } \\
\text { signals }\end{array}$ & Oscillation & Oscillation & No Oscillation \\
\hline Discontinuity & $\begin{array}{c}\text { No Disconti- } \\
\text { nuity }\end{array}$ & $\begin{array}{c}\text { Discontinue at } \\
x=0\end{array}$ & $\begin{array}{c}\text { Discontinue } \\
\text { at }(x, y)=(0,0) \\
\text { or } \rho=0 .\end{array}$ \\
\hline
\end{tabular}

Moreover, unlike with the sigma-transformed discontinuous control in which the control signals can blow up when the states of the forklift are close to the discontinuous point, the navigationvariables-based control is bounded. This boundedness of the control signals is due to the function of $\tan ^{-1}$ in (76), which makes the control signal $v_{2}$ bounded, $v_{2}(\rho, \varphi, \alpha) \in$ $(-\pi / 2, \pi / 2)$ for any argument of $\rho \in[0, \infty), \varphi, \alpha \in[-\pi, \pi)$.

The comparison results of the three control methods that are the chained form time varying, the sigma transformed discontinuous control, and the navigation-variables-based discontinuous controls are summarized in Tab. 1.

\section{CONCLUSION}

In this paper, the equations of motion of a forklift consisting of kinematic and dynamic equations were derived. The control design of a forklift vehicle was divided into two stages: The kinematic-based control generated the desired linear velocity and steering angle, then the PD control algorithm was used to track the control commands. Three configuration control algorithms that are time-varying, sigma-transformed discontinuous, and navigation-variables-based controls for the forklift were investigated by assuming that the control commands can be tracked perfectly. The time-varying control provided the global asymptotical convergence from an initial to a goal configuration, however, the vehicle exhibited oscillation motion and the convergence rate was extremely slow. On the other hand, the sigma-transformed discontinuous control and navigation-variables-based control provided a fast and smooth motion. The sigma-transformed discontinuous control had discontinuity at all points along $x=0$. Using this method, the control signals can blow up if the vehicle reaches or is close to $x=0$. The navigation-variables-based control used navigation variables were not defined at the goal point $\rho=0$. However, the control signals do not blow up because of the use of the function $\tan ^{-1}$. Problems of discontinuity of the navigationvariable-based control at the goal point can be solved by driving the forklift to a check point where $\rho \neq 0$, away from the goal point, and after the forklift achieves that point, the navigationvariable-based control can be implemented and the convergence of the configuration of the forklift is assured.

This conclusion expects that engineers who apply a stabilization control algorithm on a forklift vehicle should consider whether the motion resulted from the stabilization controls is suitable with an application, for instance parking of a car, docking of a forklift, or others. They should be aware of the calculated control signal, 
especially in the discontinuous control, whether the control signals may be blown up if the feedback signals come close to the discontinuous point of the controller or not. They should also notice a condition where the designed controller cannot stabilize the concerned vehicle, and a solution that can handle the problem.

\section{REFERENCES}

1. Abbasi W., Rehman F.U., Shah I., Rauf A. (2019), Stabilizing control algorithm for nonholonomic wheeled mobile robots using adaptive integral sliding mode, International Journal of Robotics and Automation, 34(2), 1-8.

2. Aicardi M., Casalino G., Bicchi A., Balestrino A. (1995), Closed loop steering of unicycle-like vehicles via Lyapunov techniques, IEEE Robotics and Automation Magazine, 2(1), 27-35.

3. Astolfi A. (1996), Discontinuous control of nonholonomic systems, Systems Control Letters, 27, 37-45.

4. Baranowski L.M., Siwek L.M. (2018), Use of 3D simulation to design theoretical and real pipe inspection mobile robot model, Acta Mechanica et Automatica, 12(3), 232-236.

5. Brockett R.W. (1983), Differential geometric control theory asymptotic stability and feedback stabilization, MA: Birkhäuser.

6. Hashimoto W., Yamashita Y., Kobayashi K. (2019), Asymptotic stabilization of nonholonomic four-wheeled vehicle with steering limitation, IEICE Transactions on Fundamental Electronics, Communications, and Computer Sciences, E102.A (1), 227-234.

7. Hespanha J.P., Morse A.S. (1999), Stabilization of nonholonomic integrators via logic-based switching, Automatica, 35(3), 385-393

8. Kłosiński J., Janusz J. J., Nycz R. (2015), The impact of the FLC controller's settings on the precision of the positioning of a payload transferred by a mobile crane, Acta Mechanica et Automatica, 8(4), 181-184.

9. Lamiraux F., Laumond J.P. (2000), Flatness and small-time controllability of multibody mobile robots: Application to motion planning, IEEE Transactions on Automatic Control, 45(10), 1878-1881.

10. Morin P.C., Samson C. (2009), Control of nonholonomic mobile robots based on the transverse function approach, IEEE Transactions on Robotics, 25(5), 1058-1073.

11. Muralidharan V., Mahindrakar, A.D. (2014), Position stabilization and waypoint tracking control of mobile inverted pendulum robot, IEEE Transactions on Control Systems Technology, 22(6), 2360-2367.

12. Murray R.M., Sastry S.S. (1993), Nonholonomic motion Planning: Steering using Sinusoids, IEEE Transactions on Automatic Control, 38(5), 700-716.

13. Pomet J.-B., Samson C. (1994), Time-varying exponential stabilization of nonholonomic systems in power form, Proceedings of the IFAC Symposium of Robust Control Design, Rio de Janeiro, $447-452$.

14. Ryu J.C., Agrawal S.K. (2010), Planning and control of underactuated mobile manipulators using differential flatness, Autonomous Robots, 29(1), 35-52.

15. S'anchez-Torres J.D., Defoort D.M., Muñoz-Vázquez A.J. (2019), Predefined-time stabilization of a class of nonholonomic systems, International Journal of Control,

DOI: $10.1080 / 00207179.2019 .1569262$
16. Samson C. (1995). Control of chained systems application to path following and time-varying point stabilization of mobile robots, IEEE Transactions on Automatic Control, 40(1), 64-77.

17. Sankaranarayanan V. Mahindrakar A.D. (2013). Configuration constrained stabilization of a wheeled mobile robot-theory and experiment, IEEE Transactions on Control Systems Technology, 21(1), 275-280.

18. Siegwart R., Nourbakhsh I. R. (2004), Introduction to Autonomous Mobile Robot, Cambridge, MA: MIT Press.

19. Soueres P., Laumond J.P. (1996). Shortest paths synthesis for a car-like robot, IEEE Transactions on Automatic Control, 41(5), 672-688.

20. Tamba T., Hong B., Hong K.-S. (2009), A path following control of an unmanned autonomous forklift. International Journal of Control, Automation, and Systems, 7(1), 113-122.

21. Tang C. P., Miller P.T., Krovi V.N., Ryu J.-C., Agrawal S.K. (2008), Kinematic control of a nonholonomic wheeled mobile manipulator - a differential flatness approach, Proceedings of the ASME Dynamic Syst. Control Conference, Ann Arbor, Michigan, USA, 2008-2253.

22. Virgalaivan I., Lipták T., Miková, L'. (2018), Snake robot locomotion patterns for straight and curved pipe, Journal of Mechanical Engineering, 68(2), 91-104.

23. Wang Y., Miao Z., Zhong H., Pan Q. (2015), Simultaneous stabilization and tracking of nonholonomic mobile robots: A Lyapunov-based approach, IEEE Transactions on Control Systems Technology, 23(4), 1440-1450.

24. Wei S., Uthaichana K., Žefran M. DeCarlo R. (2013), Hybrid model predictive control for the stabilization of wheeled mobile robots subject to wheel slippage, IEEE Transactions on Control Systems Technology, 21(6), 2181-2193.

25. Widyotriatmo A., Hong K.-S. (2008). Decision making framework for autonomous vehicle navigation, Proceedings of the SICE Annual Conference-International Conference on Instrumentation, Control and Information Technology, Tokyo, Japan, 20-22 August, 10021007.

26. Widyotriatmo A., Hong K.-S. (2012), Switching algorithm for robust configuration control of a wheeled vehicle, Control Engineering Practice, 20(3), 315-325.

27. Widyotriatmo A., Hong K.-S. (2015), Configuration control of an autonomous vehicle under nonholonomic and field-of-view constraints, International Journal of Imaging and Robotics, 15(3), 126-139.

28. Xiao H., Li Z., Yang C., Zhang L., Yuan P., Ding, L., Wang T. (2017), Robust stabilization of a wheeled mobile robot using model predictive control based on neurodynamics optimization, IEEE Transactions on Industrial Electronics, 65(4), 3437-3446.

29. Xie X.-J., Li G.-J. (2019), Finite-time output-feedback stabilization of high-order nonholonomic systems, International Journal of Robust and Nonlinear Control, 29(9), 2695-2711.

30. Yue M.Y., Ning M. Y., Zhao X., Zong G. (2019), Point stabilization control method for WIP vehicles based on motion planning, IEEE Transactions on Industrial Informatics, 25(6), 3368-3378.

Acknowledgment: This work was supported by Indonesian Ministry of Research, Technology and Higher Education and Institut Teknologi Bandung. 